\title{
Prelacteal feeding of newborns in post-conflict Timor-Leste
}

Vishnu Khanal, $\mathrm{MPH}^{1}$, Andy H. Lee, $\mathrm{PhD}^{1 *}$, Jonia Lourenca Nunes Brites da Cruz, MHM${ }^{2}$, Rajendra Karkee, $\mathrm{MIH}^{3}$

${ }^{1}$ School of Public Health, Curtin University, Perth, Australia

${ }^{2}$ National Hospital Guido Valadares, Ministry of Health, Dili, Timor Leste

${ }^{3}$ School of Public Health and Community Medicine, BP Koirala Institute of Health Sciences, Dharan, Nepal

* Corresponding author:

Professor Andy H. Lee

School of Public Health

Curtin University

GPO Box U 1987, Perth, WA 6845, Australia

Phone: +61 $892664180 \quad$ Fax: +61 892662958

E-Mail: Andy.Lee@curtin.edu.au

Word count: 2090

Number of tables $=2$, Number of figures $=0$

Acknowledgement: The authors are grateful to Measure DHS program of ICF Macro

International for permission to use the dataset for this study.

Conflict of interest: None declared for all authors.

Source of funding: None declared for all authors. 


\begin{abstract}
Objectives: To investigate the prevalence of prelacteal feeding and its associated factors in Timor-Leste using updated data from the national survey.

Methods: Complex sample analysis was undertaken to account for the two-stage cluster design of the Demographic and Health Survey 2009-2010. Backward stepwise logistic regression was conducted to ascertain factors associated with the prevalence of prelacteal feeding.
\end{abstract}

Results: A total of 4,821 mother-infant pairs were included in the analysis. The prevalence of prelacteal feeding was $12.3 \%$ (95\% confidence interval (CI) $11.1 \%$ to $13.5 \%$ ). The most popular prelacteal food was plain water $(50.7 \%)$, followed by glucose/sugar water $(32.5 \%)$ and milk other than breastmilk (22.7\%). Older mothers (35-49 years), mothers with upper socioeconomic status, who perceived their newborns as small size, and those residing in urban areas, were about 1.5 times more likely to give prelacteal feeds, whereas women who followed religions other than Roman Catholic had twice the risk (adjusted odds ratio 1.98; 95\% CI 1.16 to 3.41$)$.

Conclusions: Antenatal and postnatal counselling sessions that promote exclusive breastfeeding and discourage prelacteal feeding are needed that specifically target these vulnerable subgroups of Timorese mothers.

Key words: breastfeeding, colostrum, nutrition, prelacteal feeds, Timor-Leste 


\section{INTRODUCTION}

Prelacteal feeds are given to newborns for various non-nutritional reasons such as clearing the throat and bowel, reducing colic and jaundice, and hydration $(1 ; 2)$. In some cultures, they are administered for ritualistic purposes (3). Prelacteal feeding is likely to affect the initiation of breastfeeding and reduce the benefits of colostrum. Unlike colostrum which is known to be highly nutritious and protective against bacterial infections (4), prelacteal foods are often indigestible, of less immunological value, and contaminated easily (5). Such contamination can lead to infantile diarrhoea and disrupt the establishment of normal flora in the gastrointestinal track (6).

The prevalence of prelacteal feeding varies between and within countries. In Asia, for example, reported rates ranged from $26 \%$ in China (7) to $73 \%$ in Vietnam (6). Identifying the determinants of prelacteal feeding is important to develop culturally sensitive programs to reduce its practice. Previous studies have found that maternal education, occupation, family wealth status, antenatal care visits and parity are associated with prelacteal feeding in Nepal, India and Vietnam $(2 ; 6 ; 8)$. However, these factors tend to be context specific and affected by culture (1).

Timor-Leste is one of the youngest countries in Asia with a population of about 1.21 million. Life expectancy at birth is marginally higher among females (64 years) than males (62 years) (9). The adult literacy rate is $56.1 \%$ (female: $50.9 \%$, male: $61.5 \%$ ) (10) The country gained independence from Indonesia in 2002 after a decade long conflict (11). The majority of its health infrastructure was destroyed during the independence conflict. A group of health workers who remained in the country re-established the health system with international support and local resources (11). In 2006, the per capita health expenditure at US\$45 was 
higher than some other Asian countries (12). However, Timor-Leste still suffers a high infant mortality (45 per 1000 live births), with high rates of stunting (53\%) and underweight (52\%) among children less than 5 years of age (13). A recent review estimated that $11.6 \%$ of child deaths in 2011 could be attributed to suboptimal breastfeeding and inappropriate infant feeding practices (14).

Evidence from other countries has shown that the cultural practice of prelacteal feeding is responsible for late initiation of breastfeeding (3), non-exclusive breastfeeding, and early cessation of breastfeeding $(7 ; 15 ; 16)$. Nevertheless, information is lacking on prelacteal feeding in post-conflict Timor-Leste. The present study investigated the prevalence of prelacteal feeding and its associated factors in Timor-Leste based on updated data from the national Demographic and Health Survey 2009-2010.

\section{MATERIALS AND METHODS}

\section{Survey design and participants}

The Timor-Leste Demographic and Health Survey (TDHS) is a two-stage cluster survey conducted every five years (13). The 2009-2010 survey included 26 domains from 13 districts. At the first stage, 455 (116 urban and 339 rural) enumeration areas were selected using probability sampling proportionate to size. The number of rural enumeration areas was higher to reflect the rural location of the population majority. At the second stage, 27 households were selected from each enumeration area (stratum) using systematic random sampling and approached in person by trained enumerators. A total of 11,463 households responded to the survey yielding a response rate of $98.2 \%$ and women's response rate of $95.2 \%(11)$ 
Demographic and Health Surveys have been conducted in more than 50 countries using validated methodology and reliable questionnaires. In the present TDHS, the questionnaires were translated into local languages (Tetum and Bahasa) and pretested to ensure cultural appropriateness, before administered via face-to-face interviews by trained enumerators. All the child health related variables were included for births that occurred five years $(n=9806)$ preceding the survey. The collection of information through maternal recall was similarly adopted in previous studies $(2 ; 17 ; 18)$. The de-identified dataset was publicly available (19). This study focused on 4,821 mother-children pairs who had: (i) singleton birth; (ii) aged $\leq 36$ months (to minimise recall bias); (iii) information on prelacteal feeding; and (iv) the youngest child (to avoid selection of children from the same parents). Verbal consent was obtained from each participant. Ethical approval of the TDHS 2009-2010 was obtained from the Ethics Committee of ICF Macro International and the Timor-Leste Ministry of Health.

\section{Statistical analysis}

The binary outcome variable was derived from the TDHS question: "In the first three days after delivery, was your infant given anything to drink other than breast milk?” (13) Another question solicited information on the types of prelacteal food provided. Options were: infant formula; milk other than breast milk; plain water; sugar or glucose water, gripe water, sugarsalt water solution; fruit juice; tea infusions; coffee; honey; others.

Selection of independent variables was based on plausible factors from the literature $(2 ; 8)$. Maternal age was categorised into 3 groups: 15-19, 20-34, and 35-49 years. Maternal education and paternal education were either no education, primary, secondary, or higher. Maternal occupation was classified as: not working (household work), agriculture (employee or self-employed), professional/clerical/sales/services, and manual (skilled or unskilled). The 
frequency of antenatal care (ANC) visits was categorised into: $0,1-3$, and $\geq 4$. The place of delivery was either health facility or home. Religion was originally recorded as: Roman Catholic, Muslim, Protestant, Hindu and Muslim, but since most people follow Roman Catholic, the latter religions were grouped together. Socioeconomic status, derived from wealth quintiles of family assets, was further regrouped into: lower (40\%), middle (40\%), and upper $(20 \%)(18)$. Birth order was coded as: 1, 2-3, $\geq 4$, whereas size of the newborn as perceived by the mother was recorded as small, average or large. In addition to univariate chi-square tests, backward stepwise logistic regression was conducted to ascertain factors associated with the prevalence of prelacteal feeding, with complex sample analysis being performed to account for the two-stage cluster survey design (20).

\section{RESULTS}

Of the 4,821 children, 594 (12.3\%; $95 \%$ confidence interval (CI) $11.1 \%$ to $13.5 \%)$ were provided with prelacteal feeds. Among them, 301 (50.7\%) received plain water, 193 (32.5\%) received glucose/sugar water, $135(22.7 \%)$ received milk other than breastmilk, $60(10.1 \%)$ received infant formula, $8(1.3 \%)$ received sugar-salt water solution. Only one mother did not specify the type of prelacteal food given to her infant.

In this sample, the majority (64.7\%) of mothers were in 20-34 years age group. One third $(32.3 \%)$ of mothers had no education, while $62.3 \%$ were not working in a paid job. The vast majority (98.1\%) followed the Roman Catholic religion. Slightly more than half $(54.8 \%)$ the mothers attended at least four antenatal care visits, but only a quarter $(24.8 \%)$ of them delivered their last child in a health facility. Four in ten (41.5\%) families had lower socioeconomic status, with the majority $(76.2 \%)$ residing in rural areas. Table 1 presents the prevalence of prelacteal feeding by maternal and socio-demographic factors. It appears that a 
number of these variables were associated with the introduction of prelacteal feeds according to chi-square tests (Table 1).

Stepwise logistic regression results in Table 2 further confirmed that maternal age, religion, socioeconomic status, newborn size and residential location were significant factors affecting the prevalence of prelacteal feeding. Specifically, older mothers (35-49 years), mothers with upper socioeconomic status, who perceived their newborn as small size, and those residing in urban areas, were about 1.5 times more likely to give prelacteal feeds. Women who followed religions other than Roman Catholic increased their likelihood of administering prelacteal feeds by almost two fold (adjusted odds ratio $1.98 ; 95 \%$ CI 1.16 to 3.41 ).

\section{DISCUSSION}

The first milk of mothers contains adequate nutrition and also has immunoglobulin A, lactoferrin, enzymes, maternal antibodies, and other living microbes which protect the newborn against illnesses (21). However, the introduction of prelacteal feeds is still prevalent in many cultures $(7 ; 16 ; 22)$. This study found $12.3 \%$ of Timorese infants were given prelacteal feeds. The rate was lower than other Asian countries such as China (48.8\%) (16), Nepal (26.5\%) (2), and Vietnam (73.3\%) (6), which may be attributed to the Timorese culture which traditionally favours breastfeeding (23). Moreover, personal communications with hospital staff and the Alola Foundation has confirmed that prelacteal feeding is strictly discouraged at local hospitals. Alola, founded in 2001, is a not-for-profit non-government organization operating in Timor-Leste to improve the lives of women and children (24). The foundation has been promoting exclusive breastfeeding in hospitals with its staffs being deployed within the hospital premises. The hospitals also endorse the practice of keeping 
mother and infant together, except for medical reasons such as premature birth and treatment at intensive care unit, and oblige not to feed anything without the mothers' knowledge.

A significantly higher proportion of urban mothers (17\%) gave prelacteal feeds than rural mothers (11\%). Urban residents are more exposed to advertisement of infant formula and have access to formula and/or other milk substitutes, unlike their rural counterparts who have little choice but breastfeeding. Such rural-urban disparity is common and has been reported elsewhere, for example, in China (25) and Vietnam (6; 26). Similarly, mothers from upper socioeconomic class have access and financial capacity to purchase expensive prelacteal foods such as infant formula, honey and ghee (caramel butter), whereas breastfeeding remains the only viable option for mothers who cannot afford other means of infant feeding. Our finding is consistent with previous studies conducted in China (25) and Vietnam(6).

Mothers who perceived their newborn as smaller than average size were more at risk of introducing prelacteal feeds. Similar results have been reported in Nepal (27) and India (8). Mothers can face many problems in lactating small infants, such as poor sucking, lack of confidence to manage them, and possible separation due to premature birth, illness and hospital stay at neonatal care unit (28). Under such circumstances, mothers and other family members may find it easier to introduce other foods to the newborn instead of breast milk.

In this study, older mothers aged 35-49 years were found to be 50\% more likely to introduce prelacteal feeds than the main age group of 20-34 years, even after accounting the effects of other confounding factors. Almost all (99\%) of these older mothers had previous childbirth experience and had delivered at home ( $82 \%)$. They were thus less exposed to breastfeeding education and advice (29) from health workers or other sources to adopt healthy practices 
including the avoidance of prelacteal feeding. In any case, our finding suggests that older mothers should be targeted when enforcing breastfeeding promotion in the community.

According to the local religious value, mothers are encouraged to provide breastmilk to their newborn. However, Timorese mothers following other religions were significantly associated with the practice of prelacteal feeding. Although they reported an apparently higher prevalence (20.6\%) than the main Roman Catholic group (12.1\%), the sample size was rather small $(\mathrm{n}=100)$, so that further in-depth investigation of their preference is required. Indeed, differences in infant feeding practices between religions have also been observed in India (22).

The TDHS 2009-2010 reported that half $(51.5 \%)$ of infants aged five months and younger were being exclusively breastfed, and the mean duration of exclusive breastfeeding was only 2.5 months (13). In view of the National Nutrition Strategy Timor-Leste (2004) which stated that nutritional knowledge and education are key to reduce under-nutrition in the country (23), counselling sessions for mothers during antenatal and postnatal periods by health workers or community volunteers are necessary to reduce the prelacteal feeding practice and promote exclusive breastfeeding to the first six months of birth $(30 ; 31)$. Such programs should explicitly emphasize the lack of nutrients and harmful effects of prelacteal feeds (6).

The present study utilised the large dataset from the most recent national survey which was conducted in accordance to internationally validated methodology, while the analyses took into account the two-stage sampling design. It provides the first population-based report on prelacteal feeding and the results are generalizable to the entire country. However, several limitations should be considered when interpreting the findings. The TDHS was a cross 
sectional survey so that cause-effect relationships could not be established. Moreover, the recall time since birth to three years of age might introduce errors and pose difficulty for some mothers, even though retrospective recall methods have been adopted in similar settings $(2 ; 8)$. Residual confounding was another issue, despite main plausible confounding factors influencing prelacteal feeding from the literature have been controlled for in the multivariable stepwise logistic regression analysis.

\section{CONCLUSION}

This study found a relatively low prevalence of $12.3 \%$ prelacteal feeding in Timor-Leste. Small size infants and those born to older mothers, residing in urban areas, whose family belonging to non-Roman Catholic religion, were at risk of prelacteal feeding. Antenatal and postnatal counselling that promote breastfeeding and discourage prelacteal feeding should target these vulnerable population subgroups. 


\section{References}

1. Fikree FF, Ali TS, Durocher JM, Rahbar MH. Newborn care practices in low socioeconomic settlements of Karachi, Pakistan. Social Science \& Medicine 2005;60(5):911-921.

2. Khanal V, Adhikari M, Sauer K, Zhao Y. Factors associated with the introduction of prelacteal feeds in Nepal: findings from the Nepal demographic and health survey 2011. Int Breastfeed J 2013;8(1):9.

3. Szajewska H, Horvath A, Koletzko B, Kalisz M. Effects of brief exposure to water, breast-milk substitutes, or other liquids on the success and duration of breastfeeding: a systematic review. Acta paediatrica 2006;95(2):145-152.

4. Rogers NL, Abdi J, Moore D et al. Colostrum avoidance, prelacteal feeding and late breast-feeding initiation in rural Northern Ethiopia. Public Health Nutr 2011;14(11):2029-2036.

5. Laroia N, Sharma D. The Religious and Cultural Bases for Breastfeeding Practices Among the Hindus. Breastfeeding Medicine 2006;1(2):94-98.

6. Nguyen PH, Keithly SC, Nguyen NT et al. Prelacteal feeding practices in Vietnam: challenges and associated factors. BMC Public Health 2013;13(1):932.

7. Qiu L, Xie X, Lee A, Binns CW. Infants' first feeds in Hangzhou, PR China. Asia Pacific Journal of Clinical Nutrition 2007;16 Suppl 1:458-461.

8. Patel A, Banerjee A, Kaletwad A. Factors Associated with Prelacteal Feeding and Timely Initiation of Breastfeeding in Hospital-Delivered Infants in India. Journal of Human Lactation 2013.

9. United Nations Statistics Division. Timor-Leste: United Nations Statistics Division,, 2014; http://data.un.org/CountryProfile.aspx?crName=Timor-Leste. 
10. National Statistics Directorate (NSD), United Nations Population Fund (UNFPA). Population and Housing Census of Timor-Leste, 2010.Dili, Timor-Leste: National Statistics Directorate (NSD) and United Nations Population Fund (UNFPA), 2011; https://www.mof.gov.tl/wp-content/uploads/2011/06/Publication-2-English-Web.pdf.

11. Wild KJ. Maternity waiting homes and the shaping of maternal health policy in Timor-Leste 2011.

12. Saikia U, Hosgelen M. Timor-Leste's demographic destiny and its implications for the health sector by 2020. Journal of Population Research 2010;27(2):133-146.

13. National Statistics Directorate (NSD) [Timor-Leste], Ministry of Finance [TimorLeste], ICF Macro Internatioal Inc. Timor-Leste Demographic and Health Survey 2009-10. Dili, Timor-Leste: NSD [Timor-Leste] and ICF Macro, 2010.

14. Black RE, Victora CG, Walker SP et al. Maternal and child undernutrition and overweight in low-income and middle-income countries. Lancet 2013.

15. Perez-Escamilla R, Segura-Millan S, Canahuati J, Allen H. Prelacteal feeds are negatively associated with breast-feeding outcomes in Honduras. $J$ Nutr 1996;126(11):2765-2773.

16. Tang L, Hewitt K, Yu C. Prelacteal Feeds in China. Current Pediatric Reviews 2012;8(4):304-312.

17. Senarath U, Dibley M, Agho K. Breastfeeding practices and associated factors among children under 24 months of age in Timor-Leste. European Journal of Clinical Nutrition 2006;61(3):387-397.

18. Agho KE, Dibley MJ, Odiase JI, Ogbonmwan SM. Determinants of exclusive breastfeeding in Nigeria. BMC Pregnancy Childbirth 2011;11:2. 
19. Macro International Inc. Measure DHS: Demographic and Health Surveys. : ICF Macro, 2013; http://www.measuredhs.com/what-we-do/survey/survey-display356.cfm.

20. West BT. Statistical and methodological issues in the analysis of complex sample survey data: practical guidance for trauma researchers. J Trauma Stress 2008;21(5):440-447.

21. Lawrence R. The clinician's role in teaching proper infant feeding techniques. $J$ Pediatr 1995;126:S112-117.

22. Gupta P, Srivastava VK, Kumar V, Srivastava JP. Pre-lacteal feeding practices among newborn in urban slums of Lucknow city UP, India. Hindu 2012;370:70.76.

23. Ministry of Health Timor Leste. Timor Leste - National Nutrition Strategy, 2004.Dili, Timor-Leste: Ministry of Health Timor Leste, 2004.

24. Fundasaun Alola. Maternal and Child Health Program, Annual Report 2010.Dili, Timor-Leste: Fundasaun Alola 2010; http://www.alola.org.au/wpcontent/uploads/2011/06/Alola-MCH-2010-Annual-Report-English.pdf.

25. Qiu L, Zhao Y, Binns C et al. A cohort study of infant feeding practices in city, suburban and rural areas in Zhejiang Province, PR China. International Breastfeeding Journal 2008;3(4).

26. Duong DV, Binns CW, Lee AH. Breast-feeding initiation and exclusive breastfeeding in rural Vietnam. Public Health Nutr 2004;7(6):795-799.

27. Khanal V, Sauer K. Determinants of the Introduction of Prelacteal Feeds in Rural Nepal: A Cross-Sectional Community-Based Study. Breastfeeding Med 2013.

28. Demirci JR, Sereika SM, Bogen D. Prevalence and Predictors of Early Breastfeeding Among Late Preterm Mother-Infant Dyads. Breastfeeding Medicine 2013;8(3):277285. 
29. Neupane S, Doku D. Utilization of Postnatal Care Among Nepalese Women. Matern Child Health J 2013:1-9.

30. Jolly K, Ingram L, Khan KS et al. Systematic review of peer support for breastfeeding continuation: metaregression analysis of the effect of setting, intensity, and timing. BMJ 2012;344:d8287.

31. Lawrence RA. Promotion of Breastfeeding Intervention Trial (PROBIT) a randomized trial in the Republic of Belarus. J Pediatr 2001;139(1):164-165. 
Table 1. Prevalence of prelacteal feeding by maternal and socio-demographic factors in Timor-Leste $(\mathrm{n}=4821)$

\begin{tabular}{|c|c|c|c|}
\hline Factor & $\begin{array}{l}\text { Total } \\
\text { N }(\%)\end{array}$ & $\begin{array}{c}\text { Prelacteal feeding rate } \\
\mathrm{n}(\%)^{1}\end{array}$ & $\mathbf{P}^{2}$ \\
\hline Maternal age (years) & & & 0.002 \\
\hline $15-19$ & $182(3.5)$ & $25(15.0)$ & \\
\hline $20-34$ & $3089(64.7)$ & $341(11.0)$ & \\
\hline $35-49$ & $1550(31.8)$ & $228(14.6)$ & \\
\hline Maternal education & & & 0.048 \\
\hline No education & $1589(32.3)$ & $182(11.2)$ & \\
\hline Primary & $1384(27.7)$ & $157(11.8)$ & \\
\hline Secondary & $1765(37.5)$ & $238(12.9)$ & \\
\hline Higher & $83(2.6)$ & $17(22.5)$ & \\
\hline Paternal education & & & 0.001 \\
\hline No education & $1136(27.9)$ & $137(10.1)$ & \\
\hline Primary & $1367(27.3)$ & $173(13.2)$ & \\
\hline Secondary & $1875(38.9)$ & $243(12.1)$ & \\
\hline Higher & $235(5.9)$ & $41(19.9)$ & \\
\hline Maternal occupation & & & 0.659 \\
\hline Not working & $2901(62.3)$ & $370(12.8)$ & \\
\hline Agriculture & $1357(26.2)$ & $156(11.5)$ & \\
\hline $\begin{array}{l}\text { Professional, clerical, } \\
\text { sales, services }\end{array}$ & $504(10.4)$ & $63(11.8)$ & \\
\hline Manual & $59(1.1)$ & $5(9.7)$ & \\
\hline
\end{tabular}


care visits

0

$1-3$

$\geq 4$

Place of delivery

Health facility

Home

Religion

Roman Catholic

Others

Socioeconomic status

Lower

Middle

Upper

Sex of child

Male

Female

Birth order

1

$2-3$

$\geq 4$

Size of newborn

Small

Average
631 (13.2)

$1499(32.0)$

$2691(54.8)$

0.003

$1076(24.8)$

$3736(75.2)$

428 (11.2)

$166(15.6)$

4721 (98.1)

100 (1.9)

2133 (41.5)

$1950(39.3)$

738 (19.3)

2471 (51.5)

$2350(48.5)$

766 (15.7)

1409 (30.2)

2645 (54.1)

778 (16.1)

2793 (60.2)
22 (20.6)

$572(12.1)$

$<0.001$

132 (16.8)

203 (9.4)

$259(13.1)$

0.727

304 (12.5)

$290(12.1)$

0.093

$106(13.3)$

$149(10.5)$

$339(13.0)$

$<0.001$

$130(16.8)$

0.021$$
0.001
$$

$<0.001$

$313(10.8)$ 
Large

Frequency of reading

newspaper/magazine

Not at all

Less than once a week

At least once a week

Almost everyday

Frequency of watching

\section{television}

Not at all

Less than once a week

At least once a week

Almost everyday

Residential location

Urban

Rural

$146(13.8)$

1155 (23.7)

0.114

3447 (63.9)

397 (11.9)

709 (15.3)

$91(11.4)$

$575(13.1)$

$88(13.7)$

89 (2.3)

$18(21.4)$

$<0.001$

$3136(61.6)$

$348(11.1)$

$410(8.2)$

$41(9.0)$

359 (7.6)

$58(14.9)$

$916(22.6)$

$147(15.9)$

$<0.001$

1085 (23.8)

$184(16.9)$

3736 (76.2)

410 (10.9)

${ }^{1}$ From complex sample analysis; ${ }^{2}$ Chi-square test of association 
Table 2. Factors associated with prelacteal feeding in Timor-Leste $(n=4821)$

\begin{tabular}{lcc}
\hline Factor & Crude odds ratio & Adjusted odds ratio $^{1}$ \\
& $(95 \%$ confidence interval $)$ & $(95 \%$ confidence interval $)$
\end{tabular}

Maternal age (years)

20-34

$15-19$

$35-49$

Socioeconomic status

Lower

Middle

Upper

Size of newborn

Average

Small

Large

Residential location

Rural

Urban

\section{Religion}

Roman Catholic

Others
1.00

1.00
$1.57(0.99,2.47)$

$1.46(1.20,1.77)$

$\mathrm{P}=0.003$

1.00

$1.43(1.12,1.82)$

$1.66(1.21,2.27)$

$\mathrm{P}=0.001$

1.00

1.00

$1.67(1.27,2.20)$

$1.25(0.97,1.62)$

$\mathrm{P}=0.021$

1.00

1.00

$1.67(1.29,2.16)$

$1.39(1.05,1.83)$

$\mathrm{P}=0.014$

1.00

1.00

${ }^{1}$ From backward stepwise logistic regression model with complex sample analysis; variables excluded were maternal education, paternal education, maternal occupation, frequency of antenatal visits, place of delivery, sex of child, birth order, frequency of reading newspaper/magazine, frequency of watching television. 